\section{Automated Particle Analysis by Microscopy, A Primer}

Tim B. Vander Wood, MVA, Inc.

Questions about samples consisting entirely of a collection of microparticles come from people involved in a wide variety of industries dealing with powders, from pharmaceuticals to industrial minerals, powdered coatings to industrial hygiene concerns. The questions often asked are morphological. How big? How long? How wide?, and often pose a special problem for the microscopist. The time and tedium associated with answering these questions for a significant subset of the sample particles without an automated system causes the cost to skyrocket and sends the requester off to the light scatterers who at least give cheap answers, never mind that nobody knows what they really mean (What is the average size of a particle with an aspect ratio of 5 ? And did you calibrate your scattering system for my particles in my dispersant or are you still using latex spheres in water?). A common additional question is "How many are quart, or titania, or organic, or...?" The inherent advantages of microscopy--the ability to determine detailed morphology in two dimensions (and maybe three), and to discriminate different particle types in a mixture--were usually overwhelmed by the cost and time involved

The situation has changed with the full integration of computers with microscopes of all kinds. No longer is it necessary for the microscopist to manually select, size and characterize individual particles; the acquisition of images in digitized form allows a properly programmed and interfaced computer to acquire digital images, search the acquired images for particles, measure and tabulate any morphological parameter that can be described in two dimensions (and in more sophisticated systems to collect analytical data e.g., color, elemental composition, for each particle in order to determine its identity). Commercial systems are available for light microscopes, SEMs, STEMs and TEMs equipped with digitizing recording systems and range from

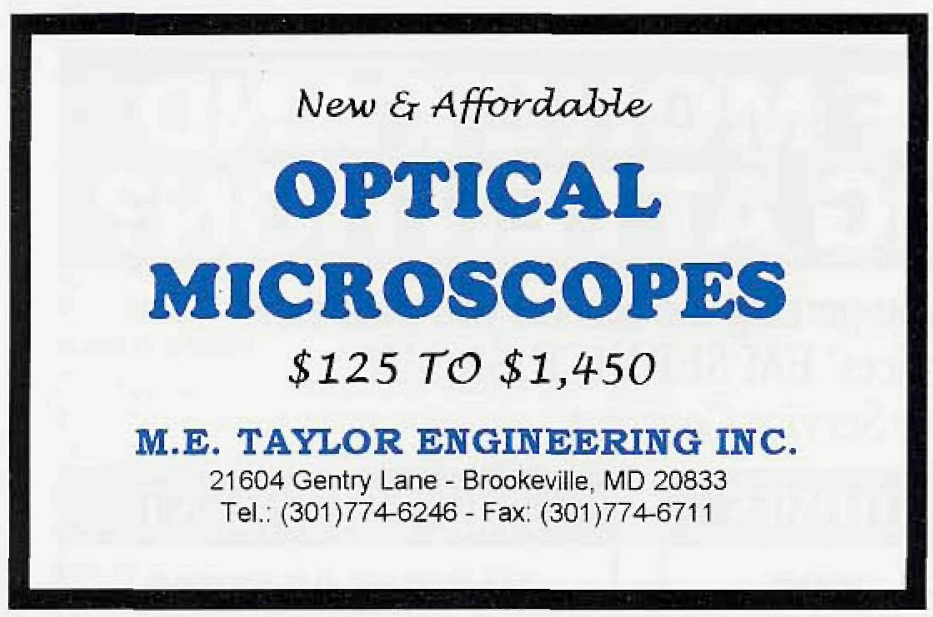

Small World Introduces:
Electron Flight Simulator a monte carlo modeling program for Windows! Electron Flight
Simulator models the interaction between the beam and your sample. Model
films, bulks and even films on a substrate! Model up to $89^{\circ}$ tilt and $400 \mathrm{KeV}$
accelerating voltage! Complex chemistries are easy, just type in the chemical
formula! Interaction volume models can be saved as Bitmap images for use
in reports! Great for teaching X-ray analysis. A must for semiconductor and
other thin film applications.
Comes with on screen periodic table and Chemistry Calculator ${ }^{\mathrm{TM}}$, the quick
way to get mean atomic weight and density values for any chemical
compound.
Call or Fax to get a free demo disk. Also ask about X-CHECKER ${ }^{\mathrm{TM}}$
calibration standard for SEM/EDS systems.
Small World, P.O. Box 25284 San Mateo, CA 94402 Phone/Fax (415) 345-8013

those you can assemble from various components at modest cost to fully integrated systems from a single vendor. All of these allow the characterization of hundreds to thousands of individual particles in a short time (generally less than an hour) with minimal involvement while the analysis is underway.

To perform an automated particle analysis you need a microscope, a digitizing imager, a computer interface to the microscope and imager, and software to analyze the digital image and report the results. A motorized sample stage is nice but not essential. Integrated systems for light microscopy are available from microscope manufacturers and companies specializing in image analysis. For electron microscopy, all major EDS manufacturers offer sophisticated packages combining computer control of the SEM (or STEM), image acquisition and EDS analysis of identified particles. If you have more time than money you may want to put a system together from off-the-shelf parts, paying the price in time to integrate them. If your budget will handle it, purchase of a commercial integrated system will save a lot of aggravation.

The digital imaging system you use will vary with your application and budget. For a light microscope, a video camera and frame grabber board combination is used. Several formats are available, and you must be careful to match camera, board and software. Although high end systems of very high resolution are available, for many purposes standard NTSC video will be adequate. The resulting digitized images can be stored for later analysis or fed directly to the image analysis program. The ability to digitize, store and analyze color images (as opposed to grayscale) is useful, but adds significantly to the cost. A similar system can be used for TEMs, but the availability of suitable cameras is limited and the cameras are expensive. The requirement for interfacing to the SEM (and STEM) scan generator limits the options for do-it-yourself systems on these microscopes. In addition to the major EDS manufacturers, at least two aftermarket companies offer systems that use your own computer to interface to scanning microscopes and acquire digitized images. If you have a tight budget with only occasional need, you can use a flatbed scanner (available for about $\$ 12 /$ hour at your local Kinko's) to digitize a photomicrograph for later image analysis and particle size and morphology measurement

If you are putting together a system from scratch, you will be well served to begin with a computer that was designed from the start to handle images. This choice of the Macintosh also allows you to use an excellently priced (free!, I downloaded mine from an Internet service) image analysis package. NIH Image. Commercial image analysis systems are built mostly on or for PC's, and occasionally on workstations (The reason for this, as one vendor admitted in an unguarded moment, is that it is hard to beat the price/performance ratio of NIH Image on the Mac.) The commercial software packages generally include some sort of data handling program for analyzing and reporting, or may include a spreadsheet in the bundle with custom written macros to automate the most common tasks. If you are putting together your own system, you will need to buy a spreadsheet and write some macros. If you enjoy inventing interpretations out of thin air, statistical packages incorporating cluster analysis and factor analysis are for you.

If the automated system is doing all this work, what's left for the microscopist? As in all computer driven systems, the rule "garbage in, garbage out " applies, and sample preparation is critical. In particular, you must be careful to produce a dispersion of the particles that is representative of the sample as a whole on a suitable analytical substrate. You can avoid sample fractionation by appropriate mixing and subsampling strategies, but producing a dispersion without agglomeration of the particles can be a challenge. Automated particle analysis routines are dumb in that special way all computer programs are dumb, and I've never met one that could reliably recognize a feature that is (to you) obviously two touching particles as anything other than one big particle. (A word of warning for the newcomer getting a manufacturer's demo: All particle sizing programs, including NIH Image, contain routines purporting to separate touching particles by 'erosion and dilation.' This works pretty well on spheres but rarely on complex particles. Have them try it on one of your samples.) When you are preparing an unfamiliar sample for automated analysis, a good deal of experimentation may be necessary, including variation of dispersion method (dry, filtered, droplet), dispersant (water, ethanol, freon, chloroform....), concentration, and perhaps surfactant. Your choices may be complicated by the nature of the (Continued on last page) 


\section{NO MATTER HOW YOU SLICE IT!}

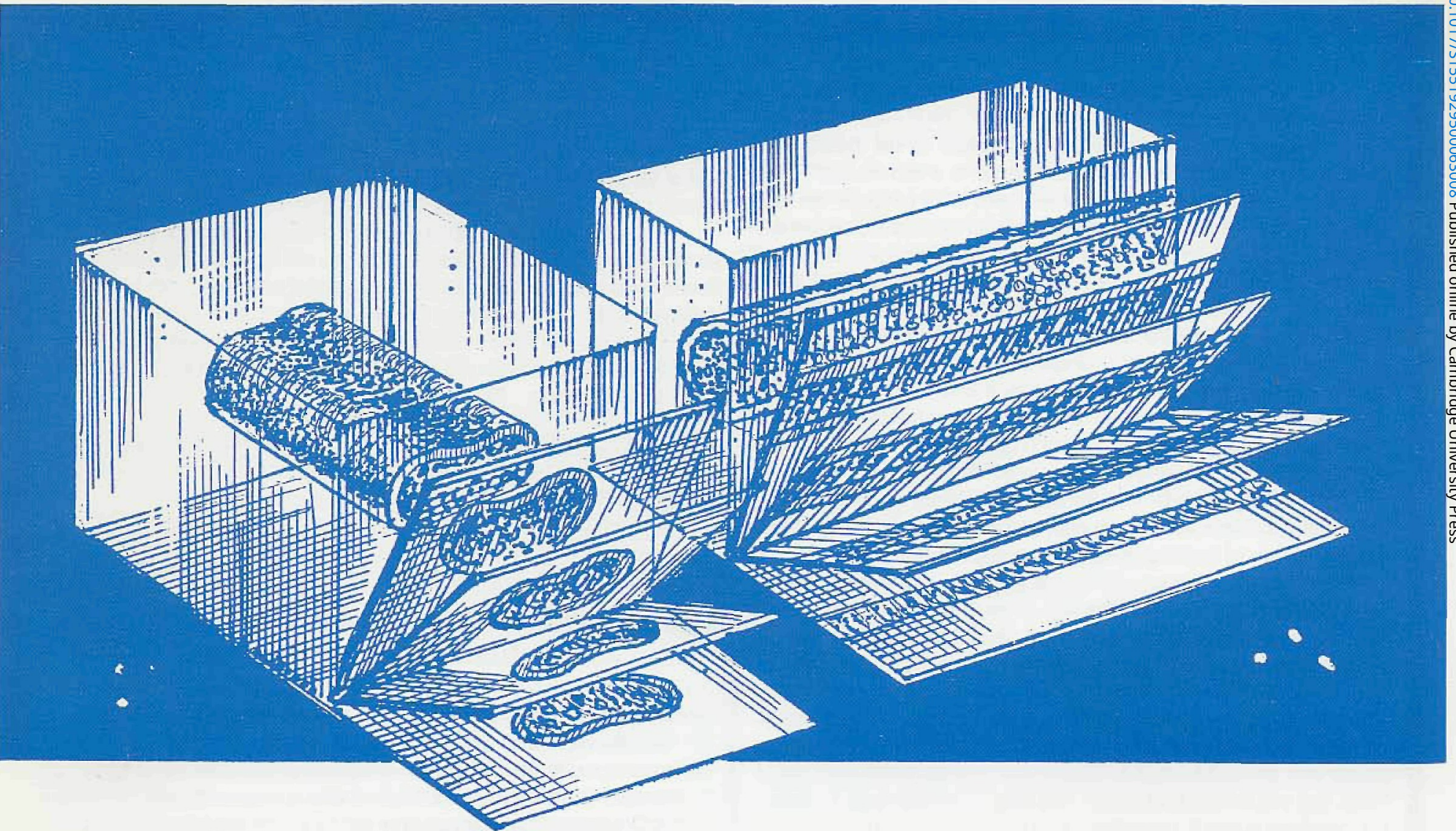

\section{You'll say that UNICRYL is a GOLDMARK idea for tissue embedding!}

\section{Here are the Features!}

\section{Its Easy and Convenient}

1. Hydrophilic acrylic resin for both LM \& EM

2. One solution - easy to use!

3. Polymerizes easily - UV or Heat

4. Provides excellent ultrastructure!

5. Preserves without chemical interaction!

6. Cleaves along tissue contour exposing antigens well!

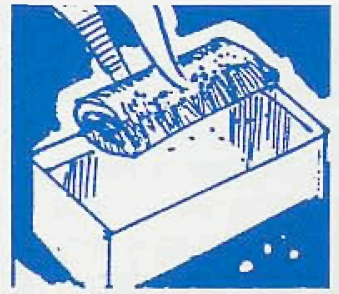

EMBED

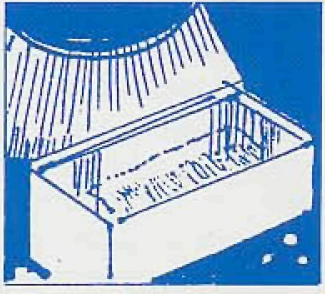

CURE

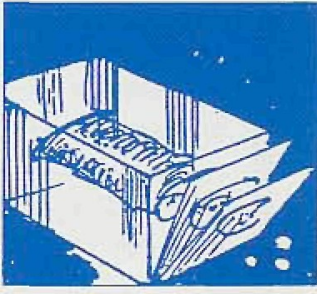

CUT

GOLDMARK BIOLOGICALS is your official British BioCell International distributor of UNICRYL, UNICRYL Staining Kit, BioMount, BioBond, and BBI colloidal gold conjugates for use in EM, LM and BL applications. We offer the best service for the lowest price. Contact us for pricing information and catalog!

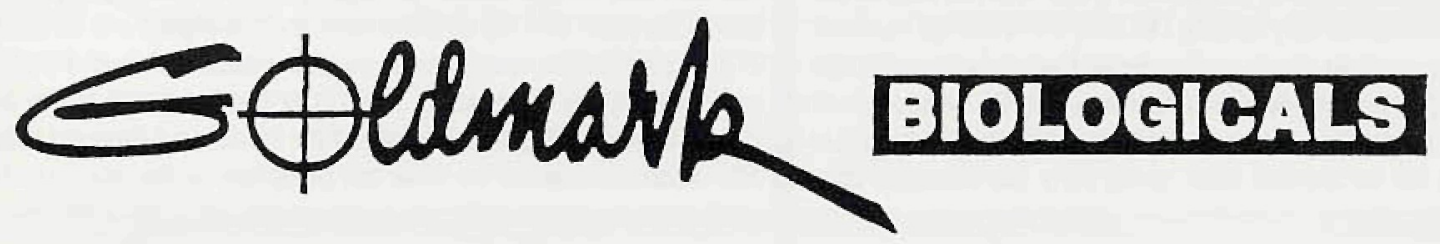




\section{Automatic Particle Analysis - Continued}

analytical substrate and its interaction with any dispersant. The high polish required of the analytical substrate (needed so that your dumb computer won't find and size substrate defects) sometimes leads to unexpected wetting behavior.

Establishing the analytical parameters is the part of the automated analysis that will seem most familiar to a microscopist. As a general rule, what looks good to you will look good to the computer, but keep in mind that the computer will recognize particles by contrast, so that any imaging or illumination mode should maximize the difference in brightness between the particles and the background without flaring the particles, which will increase their apparent size. In light microscopy, darkfield illumination is a good choice. In the SEM, backscattered electrons are often used if a suitable substrate with an atomic number much different from the particles can be found--we usually use polished carbon or aluminum, occasionally beryllium. In some cases you may want to acquire an image in which the partivcles are dark and the substrate is bright, for instance clay on gold. What matters is the difference in brightness, not the direction.

The digital image that you acquire is actually a stream of digits defining the location of each picture element (pixel) in a two dimensional grid and the intensity of the imaging signal, for example, backscattered electron current, at that pixel location. (The code that tells your computer how to assemble these numbers into an image is the "image format." PICT and TIFF are the two most common, but many others are used.) When these numbers are appropriately assembled and represented on your computer screen, you see an image, but the computer sees a two dimensional matrix, with entries representing signal intensity. Once you have this digital image, the possibilities for manipulating it are nearly unlimited. The most common adjustments are to the image brightness and contrast, which need not be linear, and can imitate any function. This step is entertaining and creative, but if you have to take it, you probably didn't prepare and setup your analysis properly

Usually the computer recognizes a particle as a set of contiguous pixels with a signal intensity above a background level which you establish. Some programs require that you first convert the image to a binary, which sets all pixels above the background to ' 1 ' and all those below to ' 0 ', others allow you to define "particles" as features within a set range of the signal intensity. Most programs work only with grayscale images with a full range of 256 , but a few are able to handle color images, which contain much more information One of the major advantages of advanced microscopy based automated particle analysis comes into play for samples that consist of a mixture of particle types. If you can differentiate different types from one another by color or brightness, then the data for each type can be compiled separately, allowing for example, the simultaneous but separate determination of iron oxide and carbon black particle morphologies in copier toner, an impossible feat for non-microscopical techniques.

Once the particles are recognized by the computer as distinct features, they can be measured. A great number of possible measurements exist, and the ones you choose will depend on why the analysis is being done in the first place. The most common measurements are maximum diameter, minimum diameter and average diameter. Besides these mundane parameters, things like particle perimeter, Feret diameters, convex area or circularity may be requested. The algorithm may also record the position of the particle. For systems integrated into the electron microscope, this ability allows EDS analyses of each particle to be done and the resulting elemental data to be stored.

All of the data you so collect is worthless if it cannot be communicated. It may be enough for you to hand a disk with raw data to the person who needs it, but its not very fulfilling. Most of the challenge involved in automated particle analysis (once you've figured out how to set it up) comes in trying to understand the data, and there is a lot of it. measurement of six morphological parameters and ten element $x$-ray intensities on two thousand particles is not an unusual case, giving thirty two thousand data points. Plotting by hand is not going to work. What does work is more automation, either through commercial spreadsheet or statistical programs which will accept the data in tabular form, or similar functions provided as part of the automation software. In either case, the common results are tables of summary descriptive statistics (mean, median, confidence interval, skewness, etc.) and histograms of the distributions of morphological features. When particle types can be differentiated on some basis, then all of these statistics and graphs are usually given separately for each particle type. This capability alone is worth the cost of the system, for there is no other way to measure the characteristics of different particle types in a mixture.

Automated particle analysis brings a new dimension of capability to the microscopist--ease of quantitation. Now you can report data for hundreds or thousands of individual particles and make statistically significant inferences about particulate samples without the tedium of hours spent accumulating the data. When you use these systems properly, the computer does what it does best--boring, repetitive chores requiring great precision, and you are freed to think about what it all means.

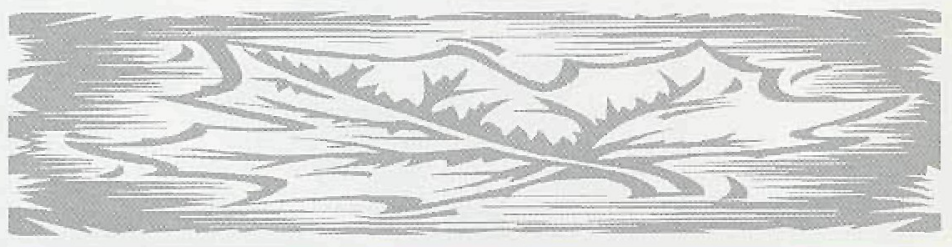
2" X 2" Projection Slides - Continued

approximately 10-20 seconds (enough time to dodge or burn in, if necessary). 5) With the room dark, except for sodium vapor lamp (which should be set with cover at darkest level), place an unexposed $2^{\prime \prime} \times 2^{\prime \prime}$ plate into position on the basebox. Expose a sufficient length of time to insure a development time of about 2 minutes. The exposure time can be determined by carrying out an exposure series on a single plate (upgraded exposures across the plate; perhaps start with $40,30,20,10 \mathrm{sec}$.). When the optimal time has been determined, expose a plate. If the electron micrograph is important, then several alternatives can be made with slight variation in exposure, with dodging (if necessary) or other effects.

6) Develop in a pan of Kodak D-19 or D-11 developer (undiluted) for 2 minutes, with continuous agitation for the first minute and no agitation thereafter. D-19 will give good contrast; use D-11 if still more contrast is needed. If you want less contrast, ty Dektol (1:2 in water).

7) Rinse plates briefly in running water, and then fix about 3-5 minutes in a pan of rapid fix (or 10 minutes in ordinary Kodak fixer).

8) Put the plates in a plastic plate holder submerged in the sink, and allow them to wash for about 30 minutes in running water. (If you prefer you can use hypo-clearing agent for 2 minutes after fixation, followed by a 10 minute wash). Dip for about 10 seconds in Photoflo (suitably diluted, according to instructions), then place the plastic plate holder in a drying oven (as dustfree as possible) for drying.

9) Place the dried plates on a light box or viewer, and select the optimal slide for each electron micrograph. Be careful not to scratch the emulsions while handling them. If you find them difficult to judge, you may want to place a temporary coverslip on the emulsion side, put them in a projector and then project them on a screen to decide.

10) Bind the final projection slides as follows: Frame the optimal field by masking with silver tape (in addition to framing the field, the masking also acts as a spacer to keep the coverglass from touching the emulsion, which might produce Newton rings). With a blower can, remove any dust from the emulsion and from one side of a clean coverglass. Put the coverglass on the emulsion side of the plate, and slip both the plate and coverglass into an Emde aluminum $2^{\prime \prime} \times 2^{n}$ slide binder, making sure that the emulsion side of the plate faces the complete side of the binder. Bend over the free edge of the binder being careful to support the adjacent side of the binder so it does not bulge out.

11) Information about the slide can be witten on the aluminum binder with a ball-point pen (which writes by engraving the soft metal surface). 


\section{If you think WDX microandysis is not for you}

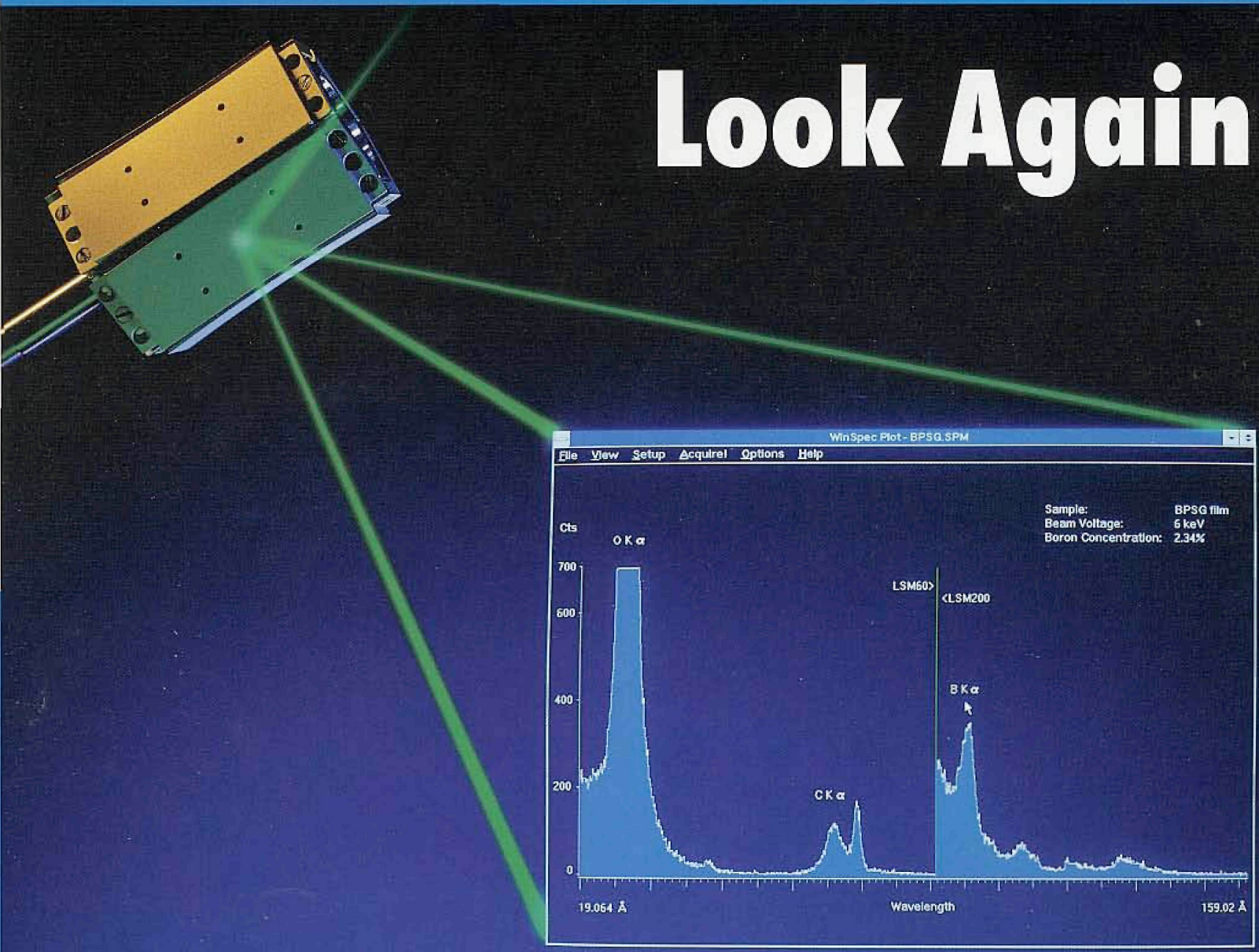

Ir's easy to get more precise resulis using the new WDX spectrometers from Microspec
It is well recognized that WDX delivers outstanding $\mathrm{X}$-ray performance for microanalysis. But you may not know how easy it can be to obtain these superior results in your lab.

With their high elemental sensitivity, accurate quantitative light-element analysis, and superb spectral resolution, the new WDX-400/600 spectrometers from Microspec allow analysis that can't be done with EDX or other analytical techniques. These systems provide the high precision needed to ensure reliable results. At the same time, the Windows ${ }^{\mathrm{TM}}$ environment makes the WDX-400/600 systems easy to use and facilitates integration into your lab. They are compatible with most SEMs and with most SEM and EDX computers. And Microspec provides full support for instrument use, with training courses and a team of service engineers.

Take a closer look at WDX. Call today for a product brochure and application notes that include direct comparisons of WDX and EDX results on samples similar to yours.

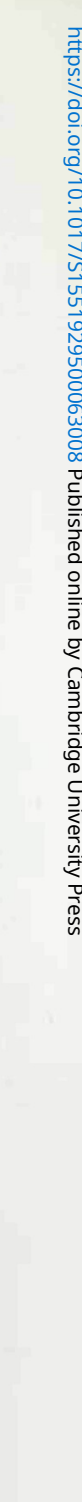




\section{IMAGINE A NEW DIMENSION}

Imagine a scanning probe microscope (SPM) that offers superior resolution and can handle samples up to eight inches in diameter. One that supports all AFM and STM scanning modes and techniques and can operate in liquid or air.

It's ideal for semiconductors magnetic media, biomaterials and other demanding samples, with integrated top-view optics and automated stepping for scanning multiple areas of your sample.

\section{Imagine Dimension ${ }^{\mathrm{TM}} 3000$,} the most versatile SPM ever manufactured.

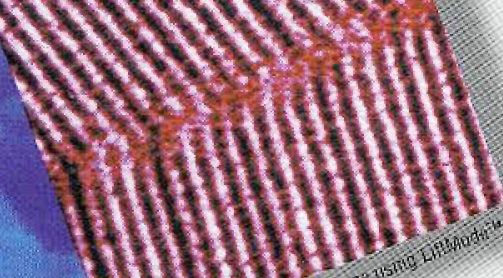

And our revolutionary new

TrakScan ${ }^{\mathrm{TM}}$ laser tracking system tracks the scanning probe tip to virtually eliminate image bow. You'll get the most accurate images and measurements possibleeasily, and at a mid-range price you can afford.

The Dimension 3000 SPMwith the NanoScope ${ }^{\circledR}$ quality you expect from the world leader in SPM.

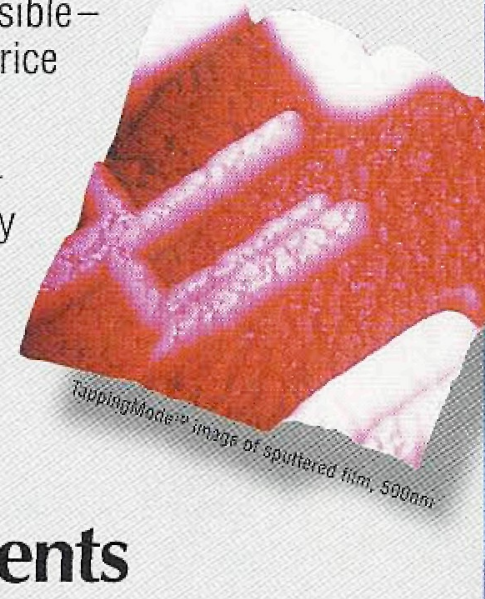

Santa Barbara, California, 800-873-9750, 805-899-3380 